PROCEEDINGS OF THE

AMERICAN MATHEMATICAL SOCIETY

Volume 129, Number 5, Pages 1437-1443

S 0002-9939(00)05937-2

Article electronically published on October 24, 2000

\title{
DUALITY OF THE WEAK ESSENTIAL NORM
}

\author{
HANS-OLAV TYLLI
}

(Communicated by Dale Alspach)

\begin{abstract}
It is established by an example that the natural quotient norms $S \mapsto \operatorname{dist}(S, W(E, F))$ and $S \mapsto \operatorname{dist}\left(S^{*}, W\left(F^{*}, E^{*}\right)\right)$ are not comparable in general. Hence there is no uniform quantitative version of Gantmacher's duality theorem for weakly compact operators in terms of the preceding weak essential norm. Above $W(E, F)$ stands for the class of weakly compact operators $E \rightarrow F$, where $E$ and $F$ are Banach spaces. The counterexample is based on a renorming construction related to weakly compact approximation properties that is applied to the Johnson-Lindenstrauss space $J L$.
\end{abstract}

\section{INTRODUCTION}

Suppose that $E$ and $F$ are Banach spaces, and let $\mathcal{L}(E, F)$ stand for the class of bounded linear operators $E \rightarrow F$. A bounded operator $S \in \mathcal{L}(E, F)$ is weakly compact if the image $S B_{E}$ of the closed unit ball $B_{E}=\{x \in E:\|x\| \leq 1\}$ is relatively weakly compact in $F$. This notion has a wide range of applications. The class $W(E, F)$ consisting of the weakly compact operators $E \rightarrow F$ forms a closed subspace of $\mathcal{L}(E, F)$. A classical duality result due to V.R. Gantmacher states that $S \in W(E, F)$ if and only if its adjoint $S^{*} \in W\left(F^{*}, E^{*}\right)$.

The weak essential norm is the quotient norm

$$
\|S\|_{w}=\operatorname{dist}(S, W(E, F)), \quad S \in \mathcal{L}(E, F),
$$

in the Banach space $\mathcal{L}(E, F) / W(E, F)$. The inequality $\left\|S^{*}\right\|_{w} \leq\|S\|_{w}$ for $S \in$ $\mathcal{L}(E, F)$ is immediate from Gantmacher's theorem. It is a natural problem whether there is any converse estimate: is there a constant $c=c_{E, F}>0$ so that

$$
c\|S\|_{w} \leq\left\|S^{*}\right\|_{w}, \quad S \in \mathcal{L}(E, F) ?
$$

This question was stated (at least) in [AT2 p. 371].

This note provides an example (Theorem 1.4 below) where (0.1) does not hold. The duality argument of the construction in Section 1 resembles that of the author's negative solution [T, 2.5] of the analogous self-duality problem for the essential norm $S \mapsto \operatorname{dist}(S, K(E, F))$, where $K(E, F)$ stands for the family of compact operators from $E$ to $F$. This earlier example suggests that (0.1) cannot hold in general. However, $T$, 2.5] involves the Banach spaces constructed by G. A. Willis, where the bounded compact approximation property differs from the usual approximation property. This result has no counterpart in the present setting, and our

Received by the editors August 17, 1999.

2000 Mathematics Subject Classification. Primary 47A30, 46B20, 46B28.

(C)2000 American Mathematical Society 
example will instead be based on a Banach space introduced by W.B. Johnson and J. Lindenstrauss [JL in their study of weakly compactly generated Banach spaces.

\section{The EXAmple}

The following approximation properties will be crucial for the self-duality problem (0.1). We say that the Banach space $E$ has the outer weakly compact approximation property (abbreviated outer WAP), if there is a constant $c<\infty$ such that

$$
\inf \{\|U-V U\|: V \in W(E),\|I-V\| \leq c\}=0
$$

holds for all Banach spaces $Z$ and all weakly compact operators $U \in W(Z, E)$. Furthermore, $E$ has the inner weakly compact approximation property if there is a constant $c<\infty$ such that

$$
\inf \{\|U-U V\|: V \in W(E),\|I-V\| \leq c\}=0
$$

holds for all Banach spaces $Z$ and all weakly compact operators $U \in W(E, Z)$. Equivalently, one may require a uniform bound on $\|V\|$ for $V \in W(E)$, but (1.1) and (1.2) have some technical advantages. The constant $c$ will only play a minor role here. The preceding weakly compact approximation properties were introduced and applied by K. Astala and the author in [AT2] and [T] (the inner version). The reference $\mathrm{AM}$ ] contains another application.

The key idea suggested by [T] is to look for a special asymmetry between the inner and the outer WAP for Banach spaces and their duals. The lack of examples of Banach spaces having the outer (or the inner) WAP makes the situation quite different from the example for the essential norm. In fact, most classical nonreflexive Banach spaces fail to have the outer or the inner WAP, in contrast with the case of the standard approximation properties defined in terms of finite rank or compact operators. This is illustrated by the following facts from [AT2, Cor.3] and [T, 3.5]. Recall that a Banach space $F$ has the Schur property if every weakly convergent sequence $\left(x_{n}\right)$ in $F$ is norm convergent. $\ell^{1}$ is the simplest space having the Schur property (see [W, III.C.9]). We refer to [LT, II.5.b] for the definition of the $\mathcal{L}^{p}$-spaces.

Example 1.1. Suppose that $E$ is a $\mathcal{L}^{1}$ - or a $\mathcal{L}^{\infty}$-space. Then:

(i) $E$ has the outer WAP if and only if $E$ has the Schur property.

(ii) $E$ has the inner WAP if and only if $E^{*}$ has the Schur property.

The desired example is obtained by applying the techniques from [T] to a Banach space introduced by Johnson and Lindenstrauss [JL, Example 1] for different purposes. It appears customary, following A.N. Plichko $[\mathrm{P}$, to denote this space by $J L$ and to call it the Johnson-Lindenstrauss space. To recall its definition, fix an uncountable family $\left\{A_{\gamma}: \gamma \in \Gamma\right\}$ of infinite subsets of $\mathbf{N}$ having the property that $A_{\gamma_{1}} \cap A_{\gamma_{2}}$ is a finite set whenever $\gamma_{1} \neq \gamma_{2}$. Let $f_{\gamma}=\chi_{A_{\gamma}} \in \ell^{\infty}$ be the characteristic function of $A_{\gamma}$ for $\gamma \in \Gamma$, and denote the algebraic linear span of $c_{0} \cup\left\{f_{\gamma}: \gamma \in \Gamma\right\}$ in $\ell^{\infty}$ by $M$. Define a norm on $M$ by

$$
\left\|x+\sum_{k=1}^{n} a_{k} f_{\gamma_{k}}\right\|=\max \left\{\left\|x+\sum_{k=1}^{n} a_{k} f_{\gamma_{k}}\right\|_{\infty},\left(\sum_{k=1}^{n}\left|a_{k}\right|^{2}\right)^{1 / 2}\right\},
$$

where $x \in c_{0}$ and $\|\cdot\|_{\infty}$ is the sup-norm in $\ell^{\infty}$. One verifies from the properties of the family $\left\{A_{\gamma}: \gamma \in \Gamma\right\}$ that the above definition yields a well-defined norm on $M$. 
The Banach space $J L$ is the completion of $M$ with respect to $\|\cdot\|$. We next state the properties from [JL, Example 1] that will be needed below.

Proposition 1.2. $\quad$ (i) $J L$ contains a natural isometric copy of $c_{0}$, so that the quotient space $J L / c_{0}$ is isometric to $\ell^{2}(\Gamma)$,

(ii) $J L^{*}$ is linearly isomorphic to $\ell^{1} \oplus \ell^{2}(\Gamma)$,

(iii) if $D \subset J L$ is a weakly compact subset, then $D$ is norm-separable.

We recall here that part (i) is a direct computation [JL p. 222]. Part (ii) is seen from the identification $J L^{*} / c_{0}^{\perp}=\ell^{1}$ and the lifting property of $\ell^{1}$. Part (iii) follows from the fact that $J L^{*}$ has a countable $\mathrm{w}^{*}$-dense subset, since there is a continuous inclusion $J L \subset \ell^{\infty}$.

Remark. Several other interesting properties of the Johnson-Lindenstrauss space were established in $\mathrm{JL}$. For instance, $J L^{*}$ is weakly compactly generated, but $J L$ is not. We refer e.g. to [CG, 4.10, 4.12, 4.15, 4.19] for a list of additional properties. The application of $J L$ in the present note differs from the earlier ones.

The general ideas in $\mathrm{T}$. are based on some natural quantities measuring the deviation of operators from weak compactness, and their connection to the inner and the outer WAP. Let $E, F$ be Banach spaces and $S \in \mathcal{L}(E, F)$. Set

$$
\begin{gathered}
\gamma_{W}(S)=\inf \left\{\varepsilon>0: S B_{E} \subset U B_{Z}+\varepsilon B_{F} \text { for some } U \in W(Z, F)\right\}, \\
\beta_{W}(S)=\inf \{\varepsilon>0:\|S x\| \leq\|U x\|+\varepsilon\|x\| \text { for all } x \in E \text { and some } U \in W(E, Z)\} .
\end{gathered}
$$

The infima above are taken over all Banach spaces $Z$. The following basic properties of $\gamma_{W}$ and $\beta_{W}$ are not difficult to check (see e.g. [A, 3.7,3.11,4.1] and [T, 1.1]):

- $\gamma_{W}(S)=0$ (respectively, $\beta_{W}(S)=0$ ) if and only if $S \in W(E, F)$,

- $\gamma_{W}$ and $\beta_{W}$ are seminorms on $\mathcal{L}(E, F)$,

- $\gamma_{W}(S T) \leq \gamma_{W}(S) \gamma_{W}(T), \beta_{W}(S T) \leq \beta_{W}(S) \beta_{W}(T)$, whenever $S T$ is defined,

- $\max \left\{\gamma_{W}(S), \beta_{W}(S)\right\} \leq\|S\|_{w}, \quad S \in \mathcal{L}(E, F)$.

The quantity $\gamma_{W}$ is the Hausdorff measure of weak non-compactness, while $\beta_{W}$ was considered in $[T]$ in a general setting. Actually,

$$
\beta_{W}(S)=\gamma_{W}\left(S^{*}\right), \quad S \in \mathcal{L}(E, F),
$$

by [GST, 1.2], but the above definition of $\beta_{W}$ will be more convenient for our construction. Some further properties and applications of $\gamma_{W}$ and $\beta_{W}$ are found in $[\mathrm{A}$, [AT2 and $[\mathrm{T}$. The references $[\mathrm{CM}]$ and $[\mathrm{CMM}]$ contain interesting uses of these seminorms in interpolation theory.

In addition, we will need the following simple fact.

Lemma 1.3. Let $E$ be a Banach space and $D \subset E$ a bounded subset. Suppose that for each $\varepsilon>0$ there is a norm-separable subset $A_{\varepsilon} \subset E$ such that

$$
\sup _{x \in D} \operatorname{dist}\left(x, A_{\varepsilon}\right) \leq \varepsilon .
$$

Then $D$ is norm-separable.

We next describe the counterexample to (0.1). The renorming argument used below in Theorem 1.4 was proved in [T, 1.2] for the general setting of closed injective operator ideals, but we prefer to include a self-contained presentation that also takes into account some relevant simplifications. The direct $\ell^{2}$-sum $\left(\bigoplus_{n} X_{n}\right)_{\ell^{2}}$ of the Banach spaces $X_{n}$ consists of the sequences $\left(x_{n}\right)$, where $x_{n} \in X_{n}$ for each $n$ and $\left\|\left(x_{n}\right)\right\|^{2}=\sum_{n}\left\|x_{n}\right\|^{2}<\infty$. 
Theorem 1.4. There are equivalent norms $|\cdot|_{n}$ on $J L$ for $n \in \mathbf{N}$ and a sequence $\left(T_{n}\right) \subset \mathcal{L}(J L, Z)$ of bounded operators, where $Z=\left(\bigoplus_{n}\left(J L,|\cdot|_{n}\right)\right)_{\ell^{2}}$, such that

$$
\left\|T_{n}\right\|_{w}=1 \text { for } n \in \mathbf{N}, \quad\left\|T_{n}^{*}\right\|_{w} \rightarrow 0 \text { as } n \rightarrow \infty .
$$

Proof. Claim 1. JL fails to have the inner WAP.

For this purpose recall from Proposition 1.2(i) that $J L / c_{0}$ is isometric to $\ell^{2}(\Gamma)$, and consider the weakly compact quotient map $q: J L \rightarrow \ell^{2}(\Gamma)$. Suppose to the contrary that there is a sequence $\left(V_{n}\right)$ of weakly compact operators $J L \rightarrow J L$ such that

$$
\left\|q-q V_{n}\right\| \leq 1 / n, \quad n \in \mathbf{N}
$$

Proposition 1.2 (iii) implies that the relatively weakly compact subsets $V_{n} B_{J L}$ are norm-separable in $J L$ for each $n$. Hence $q V_{n}\left(B_{J L}\right)$ are norm-separable subsets of $\ell^{2}(\Gamma)$ for each $n$, and

$$
\sup _{x \in B_{J L}} \operatorname{dist}\left(q x, q V_{n}\left(B_{J L}\right)\right) \leq 1 / n, \quad n \in \mathbf{N},
$$

in view of (1.4). We get from Lemma 1.3 that $\overline{q B_{J L}}=B_{\ell^{2}(\Gamma)}$ is a norm-separable set, which contradicts the non-separability of $\ell^{2}(\Gamma)$. Hence

$$
c \equiv \inf \{\|q-q V\|: V \in W(J L)\}>0,
$$

and Claim 1 has been verified.

Consider next the equivalent norms

$$
|x|_{n}=\frac{c}{n}\|x\|+\|q x\|, \quad x \in J L,
$$

on $J L$ for each $n \in \mathbf{N}$. Let $S_{n}: J L \rightarrow\left(J L,|\cdot|_{n}\right)$ be the identity map for $n \in \mathbf{N}$. It follows from the definition that $\beta_{W}\left(S_{n}\right) \leq c / n$, since $q: J L \rightarrow J L / c_{0}=\ell^{2}(\Gamma)$ is a weakly compact operator.

Claim 2 .

$$
\left\|S_{n}\right\|_{w}=\inf \left\{\left\|S_{n}-V\right\|: V \text { weakly compact } J L \rightarrow\left(J L,|\cdot|_{n}\right)\right\} \geq c .
$$

Suppose to the contrary that there is $c^{\prime}<c$ and $V \in W\left(J L,\left(J L,|\cdot|_{n}\right)\right)$ satisfying

$$
\left|S_{n} x-V x\right|_{n}=\frac{c}{n}\|x-V x\|+\|q(x-V x)\| \leq c^{\prime} \quad \text { for } x \in B_{J L} .
$$

By considering $V$ as a weakly compact operator $J L \rightarrow J L$ we get from (1.6) that $\|q-q V\| \leq c^{\prime}<c$, which contradicts (1.5). This verifies Claim 2, so that

$$
\left\|S_{n}\right\|_{w} \geq c \geq n \beta_{W}\left(S_{n}\right), \quad n \in \mathbf{N}
$$

Let $Z=\left(\bigoplus_{n}\left(J L,|\cdot|_{n}\right)\right)_{\ell^{2}}$ be the direct $\ell^{2}$-sum. Also let $J_{n}$ stand for the natural embedding $\left(J L,|\cdot|_{n}\right) \rightarrow Z$ and $P_{n}$ for the natural projection of $Z$ onto $\left(J L,|\cdot|_{n}\right)$, that is, $P_{n}\left(x_{k}\right)=x_{n}$ for $\left(x_{k}\right) \in Z$. Consider $T_{n}: J L \rightarrow Z$ defined by

$$
T_{n}=\left(\left\|S_{n}\right\|_{w}\right)^{-1} J_{n} S_{n}, \quad n \in \mathbf{N} .
$$

Thus $P_{n} T_{n}=\left(\left\|S_{n}\right\|_{w}\right)^{-1} S_{n}$ for each $n$. Note that $\left\|J_{n}\right\|=\left\|P_{n}\right\|=1$ for $n \in \mathbf{N}$. Hence the submultiplicativity of the weak essential norm and $\beta_{W}$ combined with (1.7) imply that

$$
\left\|T_{n}\right\|_{w}=\left(\left\|S_{n}\right\|_{w}\right)^{-1}\left\|S_{n}\right\|_{w}=1, \quad \beta_{W}\left(T_{n}\right)=\left(\left\|S_{n}\right\|_{w}\right)^{-1} \beta_{W}\left(S_{n}\right) \leq \frac{1}{n}
$$

for each $n \in \mathbf{N}$. 
Recall next from Proposition 1.2(ii) that $J L^{*}$ is linearly isomorphic to $\ell^{1} \oplus \ell^{2}(\Gamma)$. Note that $\ell^{1}$ has the outer WAP by Example 1.1, while reflexive spaces obviously also have this property. Hence there is a constant $c^{\prime}<\infty$, depending on the linear isomorphism, so that

$$
\inf \left\{\|U-V U\|: V \in W\left(J L^{*}\right),\|I-V\| \leq c^{\prime}\right\}=0
$$

holds for each Banach space $Y$ and each weakly compact $U \in W\left(Y, J L^{*}\right)$.

Claim 3.

$$
\left\|T_{n}^{*}\right\|_{w} \leq c^{\prime} \gamma_{W}\left(T_{n}^{*}\right), \quad n \in \mathbf{N} .
$$

Inequality (1.10) follows from the easy part of a characterization [AT2, Thm. 1] of the outer WAP, but for the sake of completeness we give the argument. Fix $n$ and suppose that $\alpha>\gamma_{W}\left(T_{n}^{*}\right)$. The definition of $\gamma_{W}$ provides a Banach space $Y$ and a weakly compact operator $U: Y \rightarrow J L^{*}$, such that

$$
T_{n}^{*}\left(B_{Z^{*}}\right) \subset U B_{Y}+\alpha B_{J L^{*}} .
$$

Let $\delta>0$. In view of (1.9) there is a weakly compact $V \in W\left(J L^{*}\right)$ so that $\|U-V U\|<\delta$ and $\|I-V\| \leq c^{\prime}$. Let $x \in B_{Z^{*}}$ be arbitrary and take $y \in B_{Y}$ satisfying $\left\|T_{n}^{*} x-U y\right\| \leq \alpha$. Consequently

$$
\left\|T_{n}^{*} x-V T_{n}^{*} x\right\| \leq\|I-V\|\left\|T_{n}^{*} x-U y\right\|+\|U y-V U y\|<c^{\prime} \alpha+\delta .
$$

It follows that $\left\|T_{n}^{*}\right\|_{w} \leq c^{\prime} \alpha+\delta$, since $V T_{n}^{*}$ is a weakly compact operator $Z^{*} \rightarrow J L^{*}$. This implies Claim 3.

Finally, we combine (1.10), (1.3) and (1.8) to obtain the estimates

$$
\left\|T_{n}^{*}\right\|_{w} \leq c^{\prime} \gamma_{W}\left(T_{n}^{*}\right)=c^{\prime} \beta_{W}\left(T_{n}\right) \leq \frac{c^{\prime} c}{n} \rightarrow 0, \quad n \rightarrow \infty .
$$

This completes the proof of the theorem.

Remarks. 1. Let $E$ be a Banach space. The quotient algebra $\mathcal{C}_{w}(E)=L(E) / W(E)$ is the corresponding weak Calkin algebra. Set $E=J L \oplus Z$ and consider $U_{n}(x, y)=$ $\left(0, T_{n} x\right)$ for $n \in \mathbf{N}$ and $(x, y) \in E$. One checks that $\left\|U_{n}\right\|_{w}=\left\|T_{n}\right\|_{w}=1$ for each $n$, while $\left\|U_{n}^{*}\right\|_{w}=\left\|T_{n}^{*}\right\|_{w} \rightarrow 0$ as $n \rightarrow \infty$. Thus $U+W(E) \mapsto\left\|U^{*}\right\|_{w}$ is an incomplete submultiplicative norm on $\mathcal{C}_{w}(E)$ that is majorized by the quotient norm $\|\cdot\|_{w}$. We refer to [M1, [M2], [AT1] and T] (as well as their references) for more results about the uniqueness or non-uniqueness of the canonical algebra norm on certain quotient algebras of $\mathcal{L}(E)$.

2. Astala and the author [AT2, Thm. 4] showed that $\gamma_{W}\left(S^{*}\right)$ and $\gamma_{W}(S)$ are not uniformly comparable in general, and a similar fact also holds for $\beta_{W}$ in view of (1.3).

3. A different weakly compact approximation property was considered by $\mathrm{O}$. Reinov [R] (see also [GW] and [LNO]). Several other renorming constructions, which differ from those of [AT1, [AT2] and [T], have been applied in connection with various approximation properties. We refer to the papers by T. Figiel and W.B. Johnson [FJ] and P.G. Casazza and H. Jarchow [CJ], among others.

4. The following is a notorious problem. Let $E$ be a complex Banach space. Does the quotient spectrum in the weak Calkin algebra $\mathcal{C}_{w}(E)$ satisfy

$$
\sigma\left(S^{*}+W\left(E^{*}\right)\right)=\sigma(S+W(E)), \quad S \in \mathcal{L}(E) ?
$$

It is useful in many concrete situations to know that the weak essential norm will be uniformly preserved by a given linear isometry. It readily follows from Theorem 
1.4 that this is not always the case. There are similar examples for the essential norm (see [AT1, 3.5]).

Example 1.5. Let $Z$ and $\left(T_{n}\right) \subset \mathcal{L}(J L, Z)$ be as in Theorem 1.4. Denote $Z_{\infty}=$ $\ell^{\infty}\left(B_{Z^{*}}\right)$ and let $J: Z \rightarrow Z_{\infty}$ be the linear isometry $J x=\left(x^{*}(x)\right)_{x^{*} \in B_{Z^{*}}}$ for $x \in Z$. Then $\left\|T_{n}\right\|_{w}=1, n \in \mathbf{N}$, but $\left\|J T_{n}\right\|_{w} \rightarrow 0$ as $n \rightarrow \infty$.

Proof. A general fact [A, 5.6] implies that $\gamma_{W}\left(S^{*}\right)=\|J S\|_{w}$ for each $S \in \mathcal{L}(J L, Z)$. Hence

$$
\left\|J T_{n}\right\|_{w}=\gamma_{W}\left(T_{n}^{*}\right) \leq\left\|T_{n}^{*}\right\|_{w} \rightarrow 0, n \rightarrow \infty
$$

by Theorem 1.4.

Finally, we state for completeness two simple positive results towards (0.1). Part (i) is well known but useful. For instance, the condition is satisfied if $F=L^{1}(0,1)$ or if $F$ is a dual space.

Proposition 1.6. Suppose that $E$ and $F$ are Banach spaces.

(i) If there is a bounded linear projection $P: F^{* *} \rightarrow F$, then

$$
\|S\|_{w} \leq\|P\|\left\|S^{*}\right\|_{w}, \quad S \in \mathcal{L}(E, F),
$$

holds for any Banach space $E$.

(ii) If $E$ has the inner WAP with constant $c$, then

$$
\|S\|_{w} \leq c\left\|S^{*}\right\|_{w}, \quad S \in \mathcal{L}(E, F),
$$

holds for any Banach space $F$.

Proof. (i) Suppose that $\delta>\left\|S^{*}\right\|_{w}$ and take a weakly compact operator $U \in$ $W\left(F^{*}, E^{*}\right)$ satisfying $\left\|S^{*}-U\right\|<\delta$. Hence

$$
\left\|S-P U_{\mid E}^{*}\right\|=\left\|P S_{\mid E}^{* *}-P U_{\mid E}^{*}\right\| \leq\|P\| \delta
$$

for $S \in \mathcal{L}(E, F)$. This yields the claim, since $P U^{*}{ }_{\mid E} \in W(E, F)$ by Gantmacher's theorem.

(ii) Assume that $E$ has the inner WAP with constant $c<\infty$ as in (1.2). It follows from the proof of [T, Thm. 1.2] and (1.3) that

$$
\|S\|_{w} \leq c \beta_{W}(S)=c \gamma_{W}\left(S^{*}\right) \leq c\left\|S^{*}\right\|_{w}
$$

for $S \in \mathcal{L}(E, F)$.

\section{REFERENCES}

[AM] A.G. Aksoy and L. Maligranda, Real interpolation and measure of weak noncompactness, Math. Nachr. 175 (1995), 5-12. MR 96m:46138

[A] K. Astala, On measures of noncompactness and ideal variations in Banach spaces, Ann. Acad. Sci. Fenn. Ser. A I. Math. Dissertationes 29 (1980), 1 - 42. MR 83a:46027

[AT1] K. Astala and H.-O. Tylli, On the bounded compact approximation property and measures of noncompactness, J. Funct. Anal. 70 (1987), 388-401. MR 88c:47020

[AT2] K. Astala and H.-O. Tylli, Seminorms related to weak compactness and to Tauberian operators, Math. Proc. Cambridge Phil. Soc. 107 (1990), 367-375. MR 91b:47016

[CJ] P.G. Casazza and H. Jarchow, Self-induced compactness in Banach spaces, Proc. Roy. Soc. Edinburgh Sect. A 126 (1996), 355-362. MR 97a:46010

[CG] J.M.F. Castillo and M. González, Three-space problems in Banach space theory, Lecture Notes in Mathematics 1667 (Springer-Verlag, 1997). MR 99a:46034

$[\mathrm{CMM}]$ F. Cobos, A. Manzano and A. Martinez, Interpolation theory and measures related to operator ideals, Quart. J. Math. 50 (1999), 401-416. CMP 2000:05 
[CM] F. Cobos and A. Martinez, Extreme estimates for interpolated operators by the real method, J. London Math. Soc. 60 (1999), 860-870. CMP 2000:11

[FJ] T. Figiel and W.B. Johnson, The approximation property does not imply the bounded approximation property, Proc. Amer. Math. Soc. 41 (1973), 197-200. MR 49:5782

[GST] M. González, E. Saksman and H.-O. Tylli, Representing non-weakly compact operators, Studia Math. 113 (1995), 265-282. MR 96i:47078

[GW] N. Grønbæk and G.A. Willis, Approximate identities in Banach algebras of compact operators, Canad. Math. Bull. 36 (1993), 45-53. MR 94b:46076

[JL] W.B. Johnson and J. Lindenstrauss, Some remarks on weakly compactly generated Banach spaces, Israel J. Math. 17 (1974), 219-230. MR 81g:46015

[LNO] Å. Lima, O. Nygaard and E. Oja, Isometric factorization of weakly compact operators and the approximation property, Israel J. Math. (to appear)

[LT] J. Lindenstrauss and L. Tzafriri, Classical Banach Spaces, Lecture Notes in Mathematics 338 (Springer-Verlag, 1973). MR 54:3344

[M1] M.J. Meyer, On a topological property of certain Calkin algebras, Bull. London Math. Soc. 24 (1992), 591-598. MR 93i:46081

[M2] M.J. Meyer, Lower bounds for norms on certain algebras, Illinois J. Math. 39 (1995), 567-575. MR 97g:46063

[P] A.N. Plichko, Some properties of Johnson-Lindenstrauss space, Funct. Anal. Appl. 15 (1981), 149-150. MR 82h:46021

[R] O. Reinov, How bad can a Banach space with the approximation property be? Math. Notes 33 (1983), 427-434. MR 85m:46018

[T] H.-O. Tylli, The essential norm of an operator is not self-dual, Israel J. Math. 91 (1995), 93-110. MR 96f:47017

[W] P. Wojtaszczyk, Banach spaces for analysts, Cambridge Stud. Adv. Math. 25 (Cambridge University Press, 1991). MR 93d:46001

Department of Mathematics, University of Helsinki, P. O. Box 4 (Yliopistonkatu 5), FIN-00014 University of Helsinki, Finland

E-mail address: hojtylli@cc.helsinki.fi 